\title{
EDITORIAL
}

\section{LA BIBLIOTECA VIRTUAL EN SALUD (BVS): UNA APUESTA POR LA DIFUSIÓN DE LA PRODUCCIÓN CIENTÍFICA ESPAÑOLA Y LATINOAMERICANA EN COLABORACIÓN CON LA OPS/OMS}

\author{
Jorge Veiga de Cabo \\ Biblioteca Nacional de Ciencias de la Salud. Escuela Nacional de Sanidad. Instituto de Salud Carlos III. Ministe- \\ rio de Sanidad y Consumo.
}

Es indudable que Internet constituye una herramienta de enorme utilidad para la obtención e intercambio de información científica, especialmente en el área de las ciencias de la salud. La enorme facilidad, técnica y económica, para la creación de portales de información supone una importante ventaja que no está exenta de riesgos e inconvenientes. Son dos los principales escollos, siendo el más inquietante que en muchas ocasiones desconocemos el origen y veracidad de la información, lo que en el terreno científico es realmente preocupante. En segundo lugar, la profusión de información dispersa en la red relacionada con biomedicina, se puede llegar a convertir en una dificultad añadida a la hora de alcanzar con éxito nuestras búsquedas, lo que nos obliga a emplear una enorme cantidad de tiempo aprendiendo la sistemática de cada portal para, posteriormente, tratar de homogeneizar la información obtenida.

Lo anteriormente expuesto, sin duda, ha generado cierta desconfianza entre la comunidad científica y los investigadores a la hora de manejar la información recogida a través de Internet, preocupación de la que también se han hecho eco organismos públicos nacionales e internacionales. En este sentido, la Organización Panamericana de la Salud de la Organización Mundial de la Salud (OPS/OMS), consciente de estos riesgos, pero también de las oportunidades de esta tecnología, se ha volcado en impulsar acciones que pretenden optimizar los beneficios y minimizar los efectos negativos que el desarrollo tecnológico pueda aportar a la comunidad científica. Así, a partir de 1998, tras la celebración del IV Congreso Panamericano de Información en Ciencias de la Salud, en San José, Costa Rica, OPS/OMS se comprometió a articular políticas y planes para desarrollar la Biblioteca Virtual en Salud (BVS) como una respuesta integradora a este desafío.

Desde entonces, el Centro Latinoamericano y del Caribe de Información en Ciencias de la Salud, anteriormente Biblioteca Regional de Medicina (BIREME) de la OPS/OMS (São Paulo, Brasil), coordina el proyecto para el desarrollo de la Biblioteca Virtual en Salud, concebida como una fuente de homogeneización, acceso y distribución de información, documentación y conocimiento científico-técnico en salud, empleando patrones de selección y siguiendo parámetros de rigor de calidad de fuentes y de contenido. La calidad de la BVS se garantiza a dos niveles: el primero, mediante la aplicación de pautas comunes en el diseño de estos portales, definidas por BIREME-OPS/ OMS; el segundo, por el establecimiento de unos criterios de selección que cada Centro Coordinador Nacional en cada uno de los países que desarrollan la BVS, ha de aplicar sobre los recursos de in- 
formación que se incluyan. Cada país participante, a través de su Centro Coordinador, desarrolla su propia BVS.

La Biblioteca Nacional de Ciencias de la Salud (BNCS) del Instituto de Salud Carlos III (ISCIII), consciente de la importancia que para la comunidad científica e investigadora de nuestro país tiene la difusión de la producción científica, en su papel de Centro Coordinador del proyecto en España, se encuentra desarrollando, desde 1999, la BVS de nuestro país en colaboración con BIREME (OPS/OMS). La presentación oficial tendrá lugar en las IX Jornadas de Información y Documentación en Ciencias de la Salud (24 al 26 de Septiembre en Cáceres), momento a partir del cual se encontrará disponible en fase piloto a través de Internet (www.isciii.es).

La Biblioteca Virtual en Salud permitirá acceder a cinco áreas: información científica, publicaciones electrónicas, herramientas de utilidad, localizador de información y otros componentes, dando acceso a bases de datos, catálogos colectivos, publicaciones electrónicas, noticias, herramientas de búsqueda, directorios, etc.

En su inicio, BVS-España contará con la primera versión del Indice Bibliográfico Español en Ciencias de la Salud (IBECS), proyecto en el que la BNCS se encuentra trabajando desde junio de $1999^{1}$. IBECS es una base de datos que recoge literatura indizada sobre ciencias de la salud publicada en España. Ha sido diseñada para incluir contenidos de las publicaciones periódicas de diferentes campos de las ciencias de la salud, tales como medicina (incluyendo salud pública, epidemiología y administración sanitaria), farmacia, veterinaria, psicología, odontología y enfermería. Con el desarrollo de IBECS se pretende disponer de un índice bibliográfico del contenido de las publicaciones de ciencias de la salud españolas que permita el acceso a la información, a la vez que facilite la difusión de las revistas cientí- ficas españolas, promocionándolas a nivel nacional e internacional.

Al objeto de establecer un sistema de control de calidad sobre su contenido, se ha creado un Comité Técnico encargado de establecer los criterios de evaluación basados en normas de presentación, difusión, contenido y rigor científico de las publicaciones, cuyo protocolo de evaluación contempla 44 ítems para cada una de las revistas.

El IBECS utiliza la metodología LILDBI/ DeCS desarrollada por BIREME y compatible con MEDLINE (DeCS/MeSH), al objeto de que en un futuro se puedan integrar las bases de datos IBECS y LILACS (Base de Datos de Literatura Latinoamericana de Ciencias de la Salud), lo que permitirá recoger esta selección de literatura científica latinoamericana y española en una única Base de Datos Bibliográfica LILACS- IBECS, como referente internacional de publicaciones producidas en países de habla hispana.

Otro apartado interesante de la BVS española es SciELO-España (Scientific Electronic Library Online), que constituye un modelo de publicación electrónica de revistas científicas en Internet que, además ser una herramienta que permite el acceso al texto completo de las publicaciones científicas, la podemos considerar como un procedimiento eficaz en la difusión del conocimiento científico generado en los países integrantes del proyecto. Una de las piezas clave que le ha permitido alcanzar la confianza de la comunidad científica y el reconocido prestigio como fuente de difusión ha sido, sin duda, el riguroso control que se estableció desde su inicio para garantizar la calidad de su contenido. Así, a través del Comité Técnico, se seleccionan las revistas científicas que, superado el proceso de evaluación de acuerdo a criterios preestablecidos de calidad, se consideren susceptibles de ser difundidos a través de SciELO. 
La metodología utilizada por SciELO ha sido estructurada sobre tres componentes. El primero de ellos permite el acceso a la edición integra y al texto completo de cada artículo de las revistas científicas incluidas, así como a la organización de la base de datos bibliográficos. Mediante este sistema, el lector accede a todos los números de cada título y al texto completo de los artículos tanto en formato HTML como en PDF, cuando este último se encuentre disponible. El segundo permite la búsqueda de artículos por autores, titulo, centro o por descriptores (se emplea el tesauro DeCS que es la traducción del MeSH del MEDLINE) y en el futuro operará en red permitiendo el acceso a los diferentes sitios SciELO de América Latina y del Caribe. Actualmente SciELO integra los sitios nacionales de Brasil, Chile y Cuba, y uno temático regional, SciELO Salud Pública, que reúne revistas científicas de América Latina y España especializadas en este área (www.scielosp.org). Como valor añadido, SciELO permite desde el apartado de bibliografía de cada artículo la conexión directa con las principales bases de datos bibliográficas internacionales, Medline, PubMed y LILACS si el artículo se encuentra referenciado en ellas y conecta con la publicación a texto completo si este ha sido editado anteriormente en SciELO. Mediante el tercer componente, el sistema es capaz de generar indicadores de uso a nivel de consulta o volcado de cada trabajo, así como un minucioso estudio de las citas bibliográficas, lo que se constituye como un importante elemento diferenciador de la metodología utilizada por SciELO frente a otros programas de edición a través de Internet.

Las pantallas de búsqueda, tanto de SciELO como de IBECS, podrán ser consultadas en español, inglés o portugués. Igualmente el tesauro estará disponible en estos tres idiomas para dar mayor facilidad de acceso y difusión a los científicos de todo el mundo. El hecho de que los diferentes sitios SciELO utilicen la misma metodología permite que la información aparezca estructurada siempre de la misma manera, facilitando el manejo y la búsqueda a los usuarios. Al objeto de posibilitar el acceso a la colección completa de revistas y recuperar el texto íntegro de los artículos, la búsqueda bibliográfica puede realizarse mediante distintos índices alfabéticos (autores, títulos, materias) y mediante formularios normalizados que amplían las posibilidades de búsqueda.

Destaca en la BVS-España la incorporación del Catálogo Colectivo de Publicaciones Periódicas en Bibliotecas de Ciencias de la Salud Españolas (C17) y de varios Directorios de utilidad científica.

El Catálogo Colectivo de Publicaciones Periódicas de las Bibliotecas de Ciencias de la Salud Españolas, denominado C-17 por contener información referente a 18.000 títulos y 145.000 colecciones de las bibliotecas de ciencias de la salud de las 17 Comunidades Autónomas españolas, permite el acceso a las colecciones de las 530 bibliotecas de ciencias de la salud españolas pertenecientes a Hospitales, Universidades, Consejerías de Sanidad, Centros de Investigación y Laboratorios (de Salud Pública y Farmacéuticos) que participan en el proyecto.

Los Directorios facilitan información sobre Publicaciones Españolas (450 publicaciones científicas editadas en España, datos sobre editorial, redacción, dirección científica $\mathrm{y}$, en caso de que exista, dirección en internet de la misma), Sociedades Científicas Españolas, Cursos y Congresos.

Con su incorporación en la Biblioteca Virtual en Salud, el Instituto de Salud Carlos III, a través de la Biblioteca Nacional de Ciencias de la Salud, apuesta por la colaboración a nivel internacional en la difusión de la producción científica de calidad, española e iberoamericana, en el campo de las Ciencias de la Salud, a través de una regulación de los contenidos y una estandarización de las metodologías. Así, a partir de un Convenio de colaboración entre el ISCIII/BNCS y la OPS/OMS/BIREME, se ofrece a los científicos de todo el mundo un acceso cómodo 
y riguroso a esta literatura científica, que en muchas ocasiones no es accesible al estar escasamente representada en las Bases de Datos internacionales, en su mayoría de origen anglosajón.

\section{BIBLIOGRAFÍA}

1. Veiga de Cabo J. El Índice Bibliográfico Español en Ciencias de la Salud. Cooperación con Latinoamérica. Rev Esp Salud Pública 1999; 529-532. 